\title{
PYTHAGOREAN RATIOS IN ARITHMETIC PROGRESSION, PART I. THREE PYTHAGOREAN RATIOS
}

\author{
by JOHN LEECH $\dagger$
}

(Received 23 June, 1992)

1. Introduction. Let $\{a, b\}$ designate the Pythagorean ratio $\left(a^{2}-b^{2}\right) / 2 a b$ between the sides of a rational right angled triangle. This paper studies the circumstances in which Pythagorean ratios can occupy consecutive places in an arithmetic progression. Part I deals with sets of three such ratios, while Part II discusses sets of four ratios.

The principal result of Part $\mathrm{I}$ is that $\{a, b\}$ is the arithmetic mean of two other Pythagorean ratios, and hence is the middle term of a three term arithmetic progression, if and only if $a / b$ is the geometric mean of two Pythagorean ratios. If these last Pythagorean ratios are both equal to $a / b$, this corresponds to the trivial solution with $\left\{p^{2}-q^{2}, 2 p q\right\}$ the arithmetic mean of $\{p, q\}$ and $\{p-q, p+q\}$. Non-trivial cases are comprehended in the following extended form of the result, which is what will actually be proved.

MaIN Theorem. Suppose we have $a^{2} / b^{2}=\{p, q\}\{r, s\}$ and $c^{2} / d^{2}=\{p, q\} /\{r, s\}$, with $a^{2} / b^{2} \neq 1 \neq c^{2} / d^{2}$. Then there are four Pythagorean ratios $\left\{\alpha_{i}, \beta_{i}\right\}$ such that

$$
\begin{aligned}
& 2\{a, b\}=\left\{\alpha_{1}, \beta_{1}\right\}-\left\{\alpha_{2}, \beta_{2}\right\}=\left\{\alpha_{3}, \beta_{3}\right\}-\left\{\alpha_{4}, \beta_{4}\right\}, \\
& 2\{c, d\}=\left\{\alpha_{1}, \beta_{1}\right\}-\left\{\alpha_{3}, \beta_{3}\right\}=\left\{\alpha_{2}, \beta_{2}\right\}-\left\{\alpha_{4}, \beta_{4}\right\} .
\end{aligned}
$$

The trivial case corresponds to one of $\{a, b\},\{c, d\}$ being zero and the two expressions for the other coinciding. The converse of this theorem will be proved in the following form.

Converse Theorem. Suppose we have $2\{a, b\}=\left\{\alpha_{1}, \beta_{1}\right\}-\left\{\alpha_{2}, \beta_{2}\right\}$. Then either we have the trivial case with $2\left\{p^{2}-q^{2}, 2 p q\right\}=\{p, q\}-\{p+q, p-q\}$, or we have the entire hypothesis and conclusion of the main theorem.

Mostly we shall ignore the trivial case without explicit mention. We need not distinguish between expressibility as the sum or difference of two Pythagorean ratios, since $-\{a, b\}=\{b, a\}$; similarly with products and quotients, since $1 /\{a, b\}=\{a+b, a-b\}$. It will sometimes be convenient to write $\{a, b\}$ as $\left\{\begin{array}{l}a \\ b\end{array}\right\}$, and $\{a+b, a-b\}$ as $\{a \pm b\}$, in places where $a$ and $b$ are replaced by more elaborate expressions.

2. Background: rational distance sets. The problem of placing points in a plane so that all pairs are at exact integer or rational distances has been the subject of several papers (for references see [3, Problem D20]). The integer points on a line are at integer distances, and it is also easy to find infinite sets of points on a circle with rational distances between all pairs, for example the points $\left(\cos 4 \theta_{i}, \sin 4 \theta_{i}\right)$, where $\tan \theta_{i}$ ranges over rational numbers. Our interest therefore lies in sets of points which do not all lie on one line or circle. In particular we may seek sets which maximize $N$, the number such that, whatever line or circle we choose, it omits at least $N$ of the points.

For infinite sets the largest known value of $N$ is 4 . This can be achieved by taking two

† The author died on 28 September, 1992.

Glasgow Math. J. 35 (1993) 395-407. 
perpendicular lines, with points on one of them at distances $\pm x, \pm y$ from their intersection. Provided that $x / y$ has at least one expression as the product of two unequal Pythagorean ratios, then it has an infinity of such expressions, to each of which there correspond points on the second line at rational distances from the first four points (and also from the intersection of the lines and from one another). In this and other configurations which include an infinity of points on a line, this line will be called the axis of the configuration.

Although these configurations give the largest known value of $N$ for infinite sets, they are not the only ones to achieve this value. Others can be constructed by inversion. If we have any set of points whose distances are all rational, then we can construct another set by taking a circle centred at a point of the set and with unit or other rational radius (in fact only the square of the radius has to be rational), and inverting the configuration in this circle. It is clear that the inverse points are at rational distances from the centre of inversion. To see that they are at rational distances from one another, we notice that, given any triangle with all its sides rational, if we invert two of its vertices in a circle of rational radius centred at the third vertex, then their inverses form with the centre of inversion a similar triangle changed by a rational scale factor. If we invert our infinite configuration in a circle centred at one of the points on its axis, then the inverse configuration comprises an infinite set of points on an axis together with two pairs of points which are mirror images in the axis but which do not all lie on a line perpendicular to it.

3. Connection with the present problem. The present problem is encountered when we consider configurations which include four points lying on two lines parallel to the axis and symmetrically disposed about it. Examples of these can be constructed in the following way. Suppose we have a configuration which includes points on a perpendicular line at distances $x, y$ from the axis which are such that a circle through them touching the axis does so at a point whose distances from these points are rational. For this we must have $x / y=\{t, u\}^{2}$ as well as the infinite set of representations $x / y=\{p, q\}\{r, s\}$. If we now invert this configuration in a circle centred at this point of contact, then the former circle inverts into a line parallel to the axis, and we obtain a configuration with points on lines parallel to the axis as described. (This configuration is studied further in Section 10.)

These, however, are not the most general configurations of infinite sets with four points on lines parallel to the axis. It will be shown that there are such configurations corresponding to any pairs of Pythagorean ratios whose product is square, not necessarily the square of another Pythagorean ratio. These configurations are thus related to certain of those with points on a line perpendicular to the axis, namely where $x / y$ is square, but the relationship does not correspond to the geometrical operation of inversion.

Suppose we have a configuration comprising an infinite set of points on an axis, two points on a line parallel to the axis, and their reflections in the axis, with all distances rational. Let $v$ be the distance between the axis and each of the parallel lines, and let $w$ be the distance between the two points on one of the side lines. Since each of these points is at a rational distance from the reflection of the other, we require $w / 2 v$ to be a Pythagorean ratio. Now consider one of the infinite set of points on the axis. Since its distance from each of the off-axis points is to be rational, we require a pair of Pythagorean ratios whose sum (or difference) is $w / v$. We thus require pairs of 
Pythagorean ratios whose sum (or difference) is twice a Pythagorean ratio, that is, triads of Pythagorean ratios in arithmetic progression, the main topic of Part I of this paper.

4. Two lemmas. These form part of a convenient line of proof of our main theorem.

Lemma 1. Suppose we have $a^{2} / b^{2}=\{p, q\}\{r, s\}$ and $c^{2} / d^{2}=\{p, q\} /\{r, s\}$. Then $a, b, c, d$ satisfy relations

$$
\frac{a^{2}+b^{2}}{2 a b}=\{c, d\}\{e, f\}, \quad \frac{c^{2}+d^{2}}{2 c d}=\{a, b\}\{g, h\},
$$

where $e, f, g, h$ are integers to be determined.

Proof. We have $\{p, q\}=a c / b d$ and $\{r, s\}=a d / b c$, so $a^{2} c^{2}+b^{2} d^{2}, a^{2} d^{2}+b^{2} c^{2}$ are both squares. Their product is therefore a square also, namely

$$
\begin{aligned}
\left(a^{2} c^{2}+b^{2} d^{2}\right)\left(a^{2} d^{2}+b^{2} c^{2}\right) & =\left(a^{2} c d \pm b^{2} d c\right)^{2}+\left(a b c^{2} \mp b a d^{2}\right)^{2} \\
& =c^{2} d^{2}\left(a^{2} \pm b^{2}\right)^{2}+a^{2} b^{2}\left(c^{2} \mp d^{2}\right)^{2} \\
& =4 a^{2} b^{2} c^{2} d^{2}\left(\left(\frac{a^{2} \pm b^{2}}{2 a b}\right)^{2}+\left(\frac{c^{2} \mp d^{2}}{2 c d}\right)^{2}\right) .
\end{aligned}
$$

Thus the two ratios $\left(\left(a^{2} \pm b^{2}\right) / 2 a b\right) /\left(\left(c^{2} \mp d^{2}\right) / 2 c d\right)$ are Pythagorean, that is

$$
\frac{a^{2}+b^{2}}{2 a b}=\{c, d\}\{e, f\}, \quad \frac{c^{2}+d^{2}}{2 c d}=\{a, b\}\{g, h\}
$$

as required.

We can go further and obtain explicit expressions for $\{e, f\},\{g, h\}$. We have

$$
\begin{aligned}
\frac{a^{2}+b^{2}}{2 a b} \cdot \frac{2 c d}{c^{2}-d^{2}} & =\frac{a^{2} / b^{2}+1}{a / b} \cdot \frac{c / d}{c^{2} / d^{2}-1} \\
& =\frac{\{p, q\}\{r, s\}+1}{\{r, s\}} \cdot \frac{1}{\{p, q\} /\{r, s\}-1} \\
& =\frac{\{p, q\}\{r, s\}+1}{\{p, q\}-\{r, s\}} \\
& =\frac{\left(p^{2}-q^{2}\right)\left(r^{2}-s^{2}\right)+4 p q r s}{2 r s\left(p^{2}-q^{2}\right)-2 p q\left(r^{2}-s^{2}\right)} \\
& =\frac{(p r+q s)^{2}-(p s-q r)^{2}}{2(p r+q s)(p s-q r)} \\
& =\{p r+q s, p s-q r\} .
\end{aligned}
$$

Similarly we find

$$
\frac{a^{2}-b^{2}}{2 a b} \cdot \frac{2 c d}{c^{2}+d^{2}}=\{p r-q s, p s+q r\} .
$$


We thus have

$$
\frac{a^{2}+b^{2}}{2 a b}=\{c, d\}\{p r+q s, p s-q r\}
$$

and

$$
\frac{c^{2}+d^{2}}{2 c d}=\{a, b\}\{(p r-q s) \pm(p s+q r)\} .
$$

In fact we have the result that $\{p r+q s, p s-q r\},\{a, b\},\{c, d\},\{(p r-q s) \pm$ $(p s+q r)\}$ are four members of a cycle of five ratios $\left\{\alpha_{i}, \beta_{i}\right\}$ satisfying $\frac{a_{i}^{2}+\beta_{i}^{2}}{2 \alpha_{i} \beta_{i}}=$ $\left\{\alpha_{i-1}, \beta_{i-1}\right\}\left\{\alpha_{i+1}, \beta_{i+1}\right\}$, with subscripts reduced modulo 5 [5], but we do not need the full force of this.

Lemma 2. Suppose we have $\frac{a^{2}+b^{2}}{2 a b}=\{c, d\}\{e, f\}$. Then products such as $\left(a^{2} e^{2}+b^{2} f^{2}\right)\left(a^{2} c^{2}+b^{2} d^{2}\right)$ are square multiples of $a^{2}+b^{2}$.

Proof. The hypothesis may be written as

$$
4 c \operatorname{def}\left(a^{2}+b^{2}\right)=2 a b\left(c^{2}-d^{2}\right)\left(e^{2}-f^{2}\right)
$$

or as

$$
a^{2} b^{2}\left(c^{2}-d^{2}\right)\left(e^{2}-f^{2}\right)=2 a b c \operatorname{def}\left(a^{2}+b^{2}\right)
$$

We thus have

$$
\begin{aligned}
&\left(a^{2} e^{2}+b^{2} f^{2}\right)\left(a^{2} c^{2}+b^{2} d^{2}\right) \\
&=\left(\left(a^{2}+b^{2}\right) e^{2}-\left(e^{2}-f^{2}\right) b^{2}\right)\left(\left(a^{2}+b^{2}\right) d^{2}+\left(c^{2}-d^{2}\right) a^{2}\right) \\
&=\left(a^{2}+b^{2}\right)\left(\left(a^{2}+b^{2}\right) d^{2} e^{2}+e^{2} a^{2}\left(c^{2}-d^{2}\right)-b^{2} d^{2}\left(e^{2}-f^{2}\right)\right) \\
&-a^{2} b^{2}\left(c^{2}-d^{2}\right)\left(e^{2}-f^{2}\right) \\
&=\left(a^{2}+b^{2}\right)\left(\left(a^{2}+b^{2}\right) d^{2} e^{2}+e^{2} a^{2}\left(c^{2}-d^{2}\right)-b^{2} d^{2}\left(e^{2}-f^{2}\right)\right) \\
&-2 a b c d e f\left(a^{2}+b^{2}\right) \\
&=\left(a^{2}+b^{2}\right)\left(a^{2} d^{2} e^{2}+b^{2} d^{2} e^{2}+e^{2} a^{2} c^{2}-e^{2} a^{2} d^{2}-b^{2} d^{2} e^{2}\right. \\
&\left.+b^{2} d^{2} f^{2}-2 a b c d e f\right) \\
&=\left(a^{2}+b^{2}\right)\left(a^{2} c^{2} e^{2}+b^{2} d^{2} f^{2}-2 a b c d e f\right) \\
&=\left(a^{2}+b^{2}\right)(a c e-b d f)^{2}
\end{aligned}
$$

as required. Similar results follow if we replace $a^{2} e^{2}+b^{2} f^{2}$ by $a^{2} f^{2}+b^{2} e^{2}$, or $a^{2} c^{2}+b^{2} d^{2}$ by $a^{2} d^{2}+b^{2} c^{2}$, or if we make both replacements.

5. Proof of main theorem. We are now ready to prove our main theorem, which we now restate.

Main Theorem. Suppose we have $a^{2} / b^{2}=\{p, q\}\{r, s\}$ and $c^{2} / d^{2}=\{p, q\} /\{r, s\}$, with $a^{2} / b^{2} \neq 1 \neq c^{2} / d^{2}$. Then there are four Pythagorean ratios $\left\{\alpha_{i}, \beta_{i}\right\}$ such that

$$
\begin{aligned}
& 2\{a, b\}=\left\{\alpha_{1}, \beta_{1}\right\}-\left\{\alpha_{2}, \beta_{2}\right\}=\left\{\alpha_{3}, \beta_{3}\right\}-\left\{\alpha_{4}, \beta_{4}\right\} \\
& 2\{c, d\}=\left\{\alpha_{1}, \beta_{1}\right\}-\left\{\alpha_{2}, \beta_{3}\right\}=\left\{\alpha_{2}, \beta_{2}\right\}-\left\{\alpha_{4}, \beta_{4}\right\}
\end{aligned}
$$


Proof. Define $x, y$ by the relations

$$
x y=c^{2} d^{2}\left(a^{2}+b^{2}\right)^{2}, \quad x-y=2 a b\left(c^{2}-d^{2}\right) .
$$

From Lemma 1 we have

$$
x-y=2 c d\left(a^{2}+b^{2}\right) \frac{2 e f}{e^{2}-f^{2}},
$$

and so

$$
(x+y)^{2}=4 c^{2} d^{2}\left(a^{2}+b^{2}\right)^{2}\left(\frac{e^{2}+f^{2}}{e^{2}-f^{2}}\right)^{2},
$$

that is

$$
x+y=2 c d\left(a^{2}+b^{2}\right) \frac{e^{2}+f^{2}}{e^{2}-f^{2}},
$$

from which we obtain

$$
x=c d\left(a^{2}+b^{2}\right) \frac{e+f}{e-f}, \quad y=c d\left(a^{2}+b^{2}\right) \frac{e-f}{e+f} .
$$

We shall now show that the four sums

$$
\begin{aligned}
& \left(x \pm c d\left(a^{2}-b^{2}\right)\right)^{2}+(2 a b c d)^{2}, \\
& \left(y \pm c d\left(a^{2}-b^{2}\right)\right)^{2}+(2 a b c d)^{2}
\end{aligned}
$$

are all squares. These expressions are

$$
\begin{aligned}
& \left(c d\left(a^{2}+b^{2}\right) \frac{e+f}{e-f} \pm c d\left(a^{2}-b^{2}\right)\right)^{2}+(2 a b c d)^{2}, \\
& \left(c d\left(a^{2}+b^{2}\right) \frac{e-f}{e+f} \pm c d\left(a^{2}-b^{2}\right)\right)^{2}+(2 a b c d)^{2},
\end{aligned}
$$

which are all perfect squares if and only if so in turn are all the expressions in each braced group below:

$$
\left.\begin{array}{c}
\left(\left(a^{2}+b^{2}\right)(e+f) \pm\left(a^{2}-b^{2}\right)(e-f)\right)^{2}+(2 a b(e-f))^{2} \\
\left(\left(a^{2}+b^{2}\right)(e-f) \pm\left(a^{2}-b^{2}\right)(e+f)\right)^{2}+(2 a b(e+f))^{2}
\end{array}\right\},
$$

Since we have $a^{2} c^{2}+b^{2} d^{2}$ square, the two expressions in the last brace are both squares by Lemma 2 , and so therefore are the preceding expressions. 
We may thus write

$$
\begin{aligned}
& \left\{\alpha_{1}, \beta_{1}\right\}=\frac{x+c d\left(a^{2}-b^{2}\right)}{2 a b c d}=\frac{x}{2 a b c d}+\{a, b\}, \\
& \left\{\alpha_{2}, \beta_{2}\right\}=\frac{x-c d\left(a^{2}-b^{2}\right)}{2 a b c d}=\frac{x}{2 a b c d}-\{a, b\}, \\
& \left\{\alpha_{3}, \beta_{3}\right\}=\frac{y+c d\left(a^{2}-b^{2}\right)}{2 a b c d}=\frac{y}{2 a b c d}+\{a, b\}, \\
& \left\{\alpha_{4}, \beta_{4}\right\}=\frac{y-c d\left(a^{2}-b^{2}\right)}{2 a b c d}=\frac{y}{2 a b c d}-\{a, b\} .
\end{aligned}
$$

Since we have

$$
\frac{x-y}{2 a b c d}=\frac{2 a b\left(c^{2}-d^{2}\right)}{2 a b c d}=2\{c, d\}
$$

we obtain the required results

$$
\begin{aligned}
& 2\{a, b\}=\left\{\alpha_{1}, \beta_{1}\right\}-\left\{\alpha_{2}, \beta_{2}\right\}=\left\{\alpha_{3}, \beta_{3}\right\}-\left\{\alpha_{4}, \beta_{4}\right\} \\
& 2\{c, d\}=\left\{\alpha_{1}, \beta_{1}\right\}-\left\{\alpha_{3}, \beta_{3}\right\}=\left\{\alpha_{2}, \beta_{2}\right\}-\left\{\alpha_{4}, \beta_{4}\right\}
\end{aligned}
$$

Although our definitions of $x, y$ involve the ratios $\{a, b\},\{c, d\}$ in an asymmetric way, it is not difficult to verify that the corresponding calculation with $\{a, b\},\{c, d\}$ interchanged leads to the same representations of $2\{a, b\}, 2\{c, d\}$ as differences $\left\{\alpha_{i}, \beta_{i}\right\}-\left\{\alpha_{j}, \beta_{j}\right\}$

6. Geometrical configurations. Before going on to prove the converse of the main theorem, I describe the geometry of the associated configurations of points at rational distances. Figure 1 shows points on two perpendicular lines, with four rational right

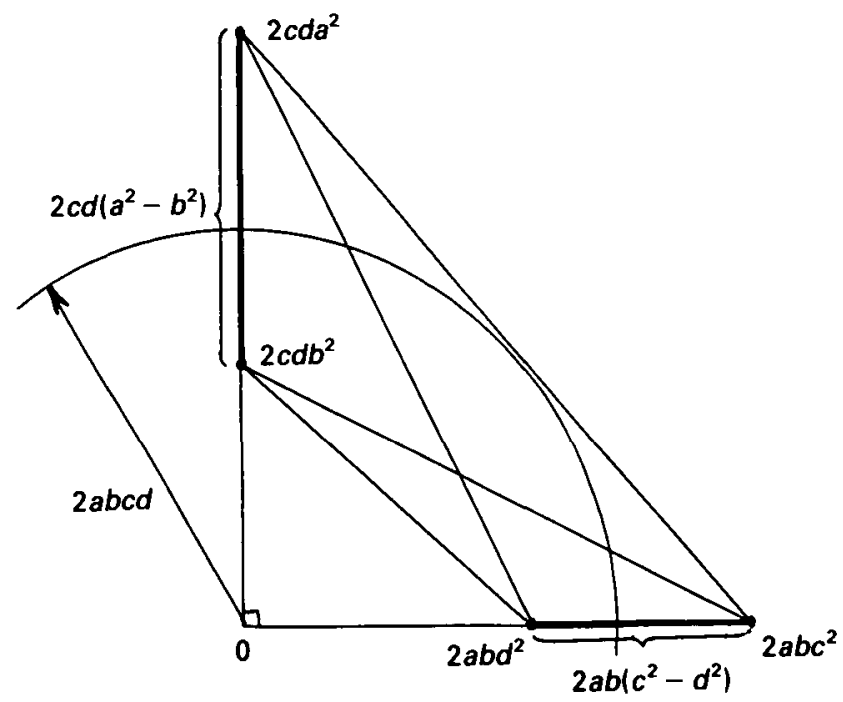

Figure 1. 


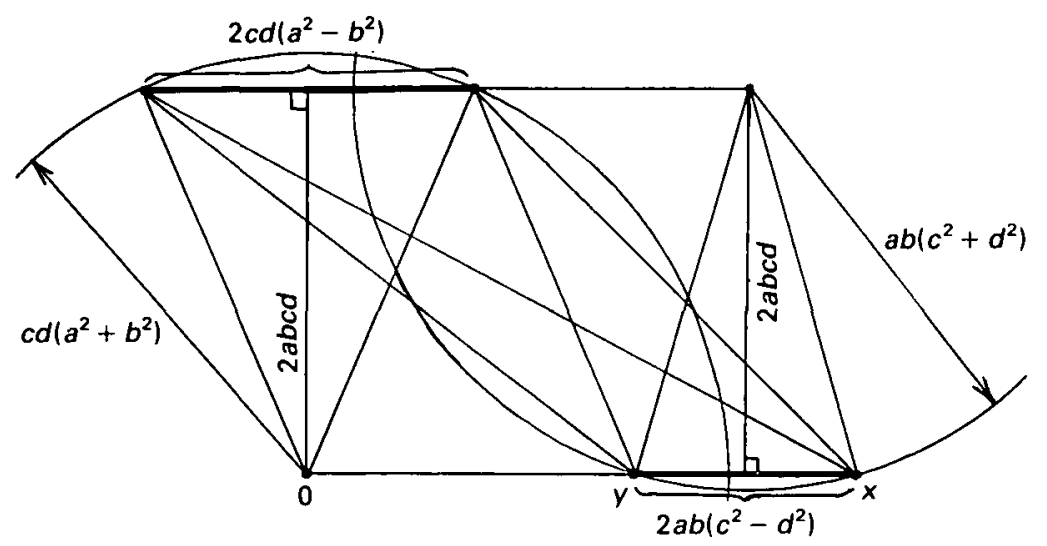

Figure 2.

angled triangles, two corresponding to each of $\{p, q\}=a c / b d,\{r, s\}=a d / b c$. The oblique joins are the hypotenuses of these triangles and so are all rational. The pairs of points are mutually inverse in the circle shown.

Figure 2 shows points on two parallel lines. The oblique joins are all rational, being the hypotenuses of rational right angled triangles corresponding to the Pythagorean ratios related as in Section 5. The pairs of points are mutually inverse in the circles shown. It is remarkable that the intervals between the points, shown by the thick lines in Figures 1, 2, are the same in the two Figures, though there is no evident relationship between their relative positions in the Figures.

In Figure 1 , the existence of a representation $a^{2} / b^{2}=\{p, q\{r, s\}$, for fixed $a / b$, implies an infinity of such representations, and so an infinity of corresponding ratios $c^{2} / d^{2}=\{p, q\} /\{r, s\}$. Thus in Figure 2 we can also fix $a / b$ and obtain an infinity of ratios $c / d$. We therefore have a configuration comprising an infinity of points on an axis and two pairs of points on lines parallel to and equidistant from the axis, being mutual reflections in the axis, with rational distances between all pairs of points of the configuration. (It is convenient for this purpose to rescale the Figures by dividing all dimensions by $2 a b c d$. We can then vary $c / d$ while keeping fixed the dimensions depending only on $a / b$.)

7. Proof of the converse theorem. We now proceed to the proof of the converse of our main theorem, in the following form.

Converse Theorem. Suppose we have $2\{a, b\}=\left\{\alpha_{1}, \beta_{1}\right\}-\left\{\alpha_{2}, \beta_{2}\right\}$. Then either we have the trivial case with $2\left\{p^{2}-q^{2}, 2 p q\right\}=\{p, q\}-\{p+q, p-q\}$, or $a / b$ is the geometric mean of two distinct Pythagorean ratios and we have the entire hypothesis and conclusion of the main theorem.

Proof. We show first that from $2\{a, b\}=\left\{\alpha_{1}, \beta_{1}\right\}-\left\{\alpha_{2}, \beta_{2}\right\}$ we can construct the whole of Figure 2, and thus we obtain the whole conclusion of the direct theorem as obtained in Section 5. Set $l / k=\{a, b\}$, so $k^{2}+l^{2}$ is square. The hypothesis of the converse theorem is that there is an $m$ such that $k^{2}+(m+l)^{2}$ and $k^{2}+(m-l)^{2}$ are both squares, so $(m+l) / k=\left\{\alpha_{1}, \beta_{1}\right\}$ and $(m-l) / k=\left\{\alpha_{2}, \beta_{2}\right\}$. (Even if $k, l$ are integers, $m$ may be only rational, and rescaling may be needed to make all quantities integers. This 
remark also applies to $n$ below.) Some subsidiary results are developed to establish the identification with Figure 2.

To locate a circle of inversion, define $n$ by the relation

$$
(n-l)(n+l)=k^{2}+(n-m)^{2},
$$

so

$$
n^{2}-l^{2}=k^{2}+n^{2}-2 n m+m^{2}
$$

and

$$
2 m n=k^{2}+l^{2}+m^{2}
$$

thus $n$ is rational. In the trivial case we have $k^{2}+l^{2}=m^{2}$ and $m=n$. We suppose hereafter that this is not the case. namely

Since $k^{2}+(m+l)^{2}$ and $k^{2}+(m-l)^{2}$ are both squares, so also is their product,

$$
\begin{aligned}
\left(k^{2}+(m+l)^{2}\right)\left(k^{2}+(m-l)^{2}\right) & =\left(k^{2}+l^{2}+m^{2}\right)^{2}-(2 l m)^{2} \\
& =(2 m n)^{2}-(2 l m)^{2} \\
& =4 m^{2}\left(n^{2}-l^{2}\right) \\
& =4 m^{2}\left(k^{2}+(n-m)^{2}\right) .
\end{aligned}
$$

Thus $(n-m) / k$ is a Pythagorean ratio, say $\{c, d\}=\left(c^{2}-d^{2}\right) / 2 c d$. We have also $m(2 n-m)=2 m n-m^{2}=k^{2}+l^{2}$, identifying the other circle of inversion. Thus the configuration of Figure 3 can be completely identified with that of Figure 2 by setting $k=2 a b c d, l=c d\left(a^{2}-b^{2}\right)$ and $n-m=a b\left(c^{2}-d^{2}\right)$. (Recall that if a triangle has all its sides rational, and two of its vertices are inverted in a circle centred at the third vertex, then the inverse points form with the centre of inversion a similar triangle. This shows that the joins in Figure 3 not already accounted for are also rational.)

In addition to $(n-m)^{2}+k^{2}$ and $k^{2}+l^{2}$ being squares, we have $(n-m)^{2}+k^{2}+l^{2}=$

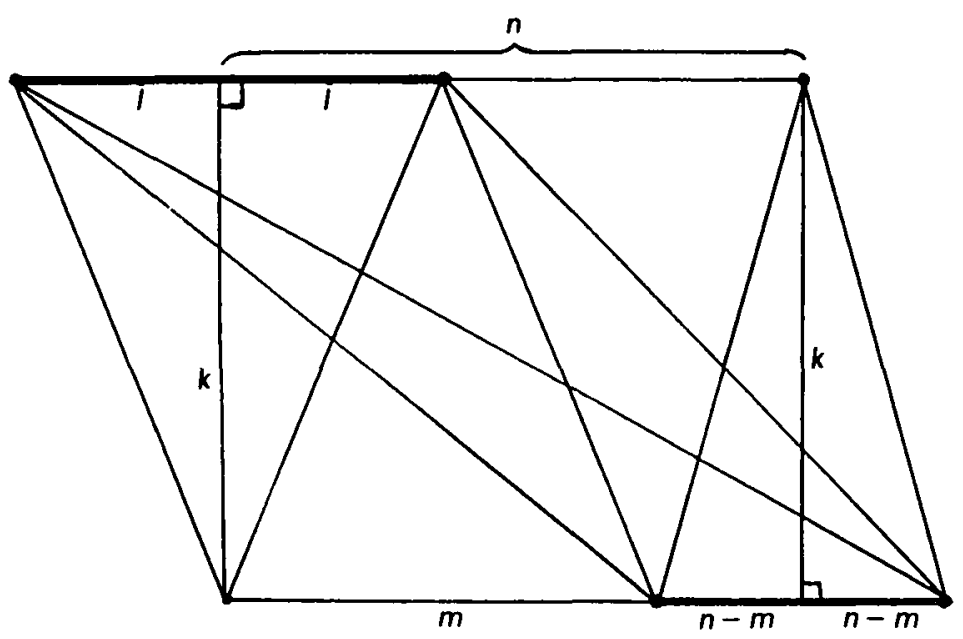

Figure 3. 
$n^{2}-l^{2}+l^{2}=n^{2}$. Thus, setting

$$
\begin{aligned}
& \{e, f\}=\frac{\sqrt{\left(k^{2}+l^{2}\right)}}{n-m}=\frac{\sqrt{\left(k^{2}+l^{2}\right)}}{k} \cdot \frac{k}{n-m}=\frac{a^{2}+b^{2}}{2 a b} /\{c, d\}, \\
& \{g, h\}=\frac{\sqrt{\left((n-m)^{2}+k^{2}\right)}}{l}=\frac{\sqrt{\left((n-m)^{2}+k^{2}\right)}}{k} \cdot \frac{k}{l}=\frac{c^{2}+d^{2}}{2 c d} /\{a, b\},
\end{aligned}
$$

we obtain

$$
\frac{a^{2}+b^{2}}{2 a b}=\{c, d\}\{e, f\}, \quad \frac{c^{2}+d^{2}}{2 c d}=\{a, b\}\{g, h\},
$$

in agreement with the conclusions of Lemma 1 and the hypothesis of Lemma 2. Thus by Lemma 2 we have that products such as $\left(a^{2} e^{2}+b^{2} f^{2}\right)\left(a^{2} c^{2}+b^{2} d^{2}\right)$ are square multiples of $a^{2}+b^{2}$.

It remains to be shown that $a^{2} c^{2}+b^{2} d^{2}$ and $a^{2} d^{2}+b^{2} c^{2}$ are themselves squares, since the converse of Lemma 1 is not valid in general. This result follows by reversing the argument in the proof of the main theorem in Section 5. With $x=2 n-m, y=m$, we now have that the sums

$$
\begin{aligned}
& \left(x^{2} \pm c d\left(a^{2}-b^{2}\right)\right)^{2}+(2 a b c d)^{2}, \\
& \left(y^{2} \pm c d\left(a^{2}-b^{2}\right)\right)^{2}+(2 a b c d)^{2}
\end{aligned}
$$

are all squares. Since we have $x y=c^{2} d^{2}\left(a^{2}+b^{2}\right)^{2}$ and $x-y=2 a b\left(c^{2}-d^{2}\right)$, as in Section 5 , we can deduce that the products $\left(a^{2}+b^{2}\right)\left(a^{2} e^{2}+b^{2} f^{2}\right)$ and $\left(a^{2}+b^{2}\right)\left(b^{2} e^{2}+a^{2} f^{2}\right)$ are squares. Combining these results with those of Lemma 2 , we deduce that $a^{2} c^{2}+b^{2} d^{2}$ and $a^{2} d^{2}+b^{2} c^{2}$ are squares. So, setting $a c / b d=\{p, q\}$ and $a d / b c=\{r, s\}$, we obtain the required results

$$
a^{2} / b^{2}=\{p, q\}\{r, s\}, \quad c^{2} / d^{2}=\{p, q\} /\{r, s\},
$$

thus completing the proof of the converse theorem.

8. Relations between triads. The sets of four triads of Pythagorean ratios, found in Section 5, may be set out in an array of the form

$$
\begin{array}{ccc}
\left\{\alpha_{1}, \beta_{1}\right\} & \{a, b\} & \left\{\beta_{2}, \alpha_{2}\right\} \\
\{c, d\} & & \{d, c\} \\
\left\{\beta_{3}, \alpha_{3}\right\} & \{b, a\} & \left\{\alpha_{4}, \beta_{4}\right\}
\end{array}
$$

in which the entries in each row and column form an arithmetic progression. Noticing that a Pythagorean ratio $\{a, b\}=\left(a^{2}-b^{2}\right) / 2 a b$ is of the form $\cot 2 \phi$, where $\tan \phi=b / a$, we examine the angles in Figure 4. Let $b / a=\tan \phi, d / c=\tan \psi, \beta_{i} / \alpha_{i}=\tan \theta_{i}$. Some of these angles are shown in Figure 4. With these angles as shown, we see that $2 \theta_{1}+2 \theta_{2}+2 \psi=\pi, 2 \theta_{3}+2 \theta_{4}-2 \psi=0$. These, however, should be interpreted with caution. Since $\theta_{i}$ is determined only by $\cot 2 \theta_{i}=\left\{\alpha_{i}, \beta_{i}\right\}, 2 \theta_{i}$ is determined only up to a multiple of $\pi$, and $\theta_{i}$ only up to a multiple of $\pi / 2$ (so $\theta_{i}$ might equally be $\arctan \left(\beta_{i} / \alpha_{i}\right)$ or $\left.\arctan \left(-\alpha_{i} / \beta_{i}\right)\right)$. We should thus write $\theta_{1}+\theta_{2}+\psi \equiv 0(\bmod \pi / 2), \theta_{3}+\theta_{4}-\psi \equiv 0$ $(\bmod \pi / 2)$. 


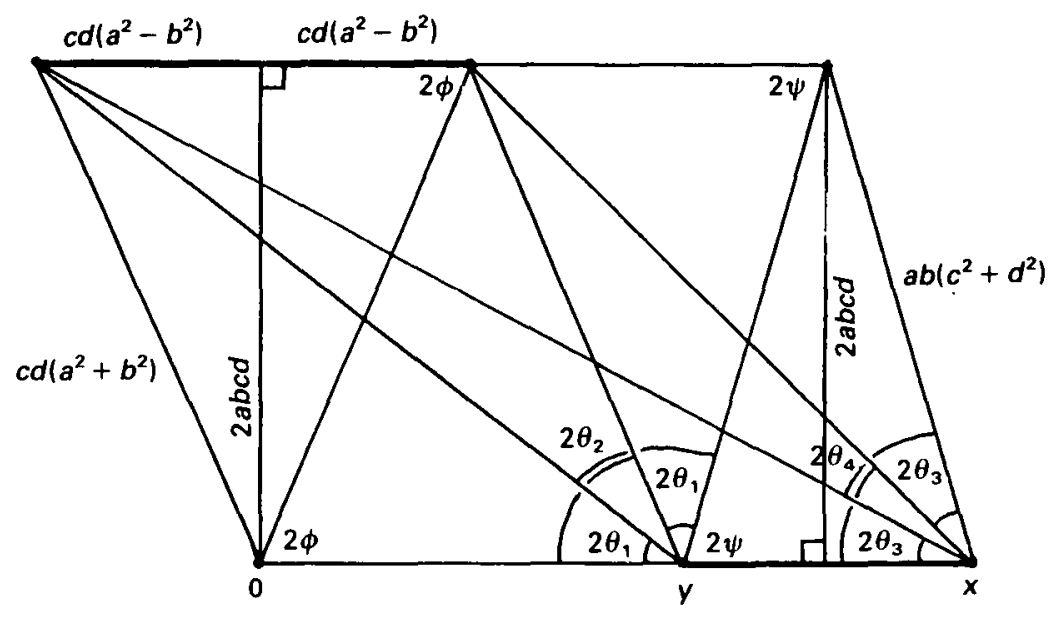

Figure 4.

With changes in notation, let us write the array of Pythagorean ratios in arithmetic progression as

$$
\begin{array}{ccc}
\cot 2 \chi_{1} & \cot 2 \phi & \cot 2 \chi_{2} \\
\cot 2 \psi & & \cot (-2 \psi) \\
\cot 2 \chi_{3} & \cot (-2 \phi) & \cot 2 \chi_{4} .
\end{array}
$$

In terms of these redefined angles, our congruences are all of the form $\chi_{1} \equiv \chi_{2}+\psi$ $(\bmod \pi / 2)$, and always relate elements in the array whose positions are equivalent to

$$
\begin{array}{ccc}
\cot 2 \chi_{1} & * & \cot 2 \chi_{2} \\
\cot 2 \psi & *
\end{array}
$$

under symmetry operations on the square array.

From these relations we can express all the Pythagorean ratios in the array in terms of those on one side of it, namely

$$
\begin{array}{ccc}
\left\{\alpha_{1}, \beta_{1}\right\} & \{a, b\} & \left\{\beta_{2}, \alpha_{2}\right\} \\
\left\{\begin{array}{l}
\alpha_{1} \alpha_{2}-\beta_{1} \beta_{2} \\
\alpha_{1} \beta_{2}+\beta_{1} \alpha_{2}
\end{array}\right\} & \left\{\begin{array}{l}
\beta_{1} \beta_{2}-\alpha_{1} \alpha_{2} \\
\beta_{1} \alpha_{2}+\alpha_{1} \beta_{2}
\end{array}\right\} \\
\left\{\begin{array}{l}
\alpha_{1} a+\beta_{1} b \\
\beta_{1} a-\alpha_{1} b
\end{array}\right\} & \{b, a\} & \left\{\begin{array}{l}
\beta_{2} a+\alpha_{2} b \\
\alpha_{2} a-\beta_{2} b
\end{array}\right\} .
\end{array}
$$

9. Some parametric examples. We have established that $\{a, b\}$ is the arithmetic mean of two Pythagorean ratios if and only if $a / b$ is the geometric mean of two Pythagorean ratios, so $a^{2} / b^{2}=\{p, q\}\{r, s\}$. This latter condition requires the right angled triangles generated by $\{p, q\}$ and $\{r, s\}$ to have equal area (up to a rational scale factor). Construction of such triangles has a long history [2], going back to Diophantus, who 
found sets of three triangles with equal area. He showed that, if we have a solution of $x^{2}+x y+y^{2}=z^{2}$ in integers, the the triangles generated by $\{z, x\},\{z, y\},\{x+y, z\}$ have equal areas. To solve $x^{2}+x y+y^{2}=z^{2}$ we take any three integers $l, m, n$ with sum zero and set $x=l^{2}-m^{2}, y=m^{2}-n^{2}$, which gives $z=-(m n+n l+l m)$. Cyclic permutation of $l, m, n$ corresponds to cyclic permutation of $x, y,-(x+y)$.

These sets of triangles of equal area lead to triads of Pythagorean ratios in arithmetic progressions of the form

$$
\begin{array}{ccc}
\left\{\begin{array}{c}
(m+n)\left(m^{2}+m n+n^{2}\right) \\
m n(m-n)
\end{array}\right\} & \left\{\begin{array}{c}
2\left(m^{2}+m n+n^{2}\right) \\
m^{2}-n^{2}
\end{array}\right\} & \{n, m\} \\
\left\{\begin{array}{l}
m(m+2 n) \\
n(2 m+n)
\end{array}\right\} & \left\{\begin{array}{l}
n(2 m+n) \\
m(m+2 n)
\end{array}\right\} \\
\left\{\begin{array}{c}
(m-n)\left(m^{2}+m n+n^{2}\right) \\
(m+n)(2 m+n)(m+2 n)
\end{array}\right\} & \left\{\begin{array}{c}
m^{2}-n^{2} \\
2\left(m^{2}+m n+n^{2}\right)
\end{array}\right\} & \left\{\begin{array}{l}
m+2 n \\
2 m+n
\end{array}\right\},
\end{array}
$$

where each row and column of three Pythagorean ratios is an arithmetic progression.

Two other simple expressions for rational right angled triangles of equal area are given by the generators

and

$$
p=2 m^{2}+n^{2}, \quad r=m^{2}+2 n^{2}, \quad q=s=m^{2}-n^{2}
$$

$$
p=2 m^{2}-n^{2}, \quad r=m^{2}-2 n^{2}, \quad q=s=m^{2}+n^{2} .
$$

The former leads to the following array of Pythagorean ratios in arithmetic progressions:

$$
\begin{aligned}
& \left\{\begin{array}{l}
m\left(2 m^{2}+n^{2}\right) \\
n\left(m^{2}+2 n^{2}\right)
\end{array}\right\} \quad\left\{2\left(m^{2}-n^{2}\right), 3 m n\right\} \quad\left\{\begin{array}{c}
3 m n\left(m^{2}-n^{2}\right) \\
\left(2 m^{2}+n^{2}\right)\left(m^{2}+2 n^{2}\right)
\end{array}\right\} \\
& \left\{\begin{array}{c}
m\left(m^{2}+2 n^{2}\right) \\
n\left(2 m^{2}+n^{2}\right)
\end{array}\right\} \\
& \{n, m\} \quad\left\{3 m n, 2\left(m^{2}-n^{2}\right)\right\} \quad\left\{3 m n, m^{2}-n^{2}\right\},
\end{aligned}
$$

and the latter to the array:

$$
\begin{aligned}
& \left\{\begin{array}{c}
m\left(2 m^{2}-n^{2}\right) \\
n\left(m^{2}+n^{2}\right)
\end{array}\right\} \quad\left\{2\left(m^{2}+n^{2}\right), 3 m n\right\} \quad\left\{3 m n, 2 m^{2}-n^{2}\right\} \\
& \left\{\begin{array}{l}
m\left(m^{2}-2 n^{2}\right) \\
n\left(2 m^{2}-n^{2}\right)
\end{array}\right\} \quad\left\{\begin{array}{l}
n\left(2 m^{2}-n^{2}\right) \\
m\left(m^{2}-2 n^{2}\right)
\end{array}\right\} \\
& \left\{\begin{array}{c}
n\left(m^{2}-2 n^{2}\right) \\
m\left(m^{2}+n^{2}\right)
\end{array}\right\} \quad\left\{3 m n, 2\left(m^{2}+n^{2}\right)\right\} \quad\left\{3 m n, m^{2}-2 n^{2}\right\} .
\end{aligned}
$$

It will be seen from the foregoing arrays that a completely general Pythagorean ratio $\{n, m\}$ can be the first (or last) member of a three term arithmetic progression in several ways, in addition to the trivial solution $\{n, m\}+\{m+n, m-n\}=2\left\{2 m n, m^{2}-n^{2}\right\}$. (The second array gives only this trivial solution in the case $m=2, n=1$.) However, not all Pythagorean ratios can occupy the middle place in a three term arithmetic progression, since it is known [1] that not all ratios $a^{2} / b^{2}$ can be expressed as products $\{p, q\}\{r, s\}$. 
For example, the integers 4 and 9 are not so expressible, so the ratios $3 / 4$ and $4 / 3$ cannot be the middle term of a three term arithmetic progression.

10. A special case. In the special case that $a^{2}+b^{2}$ is square, corresponding to three Pythagorean ratios in geometric progression, we have that $a^{2} c^{2}+b^{2} d^{2}, a^{2} d^{2}+b^{2} c^{2}$, $a^{2} e^{2}+b^{2} f^{2}$ and $a^{2} f^{2}+b^{2} e^{2}$ are all squares, by Lemma 2 . We thus see that these geometric progressions come in pairs with the same middle term $a / b$ (see [4]). The common ratios of these progressions are $c / d$ and $e / f$, which are related by

$$
\frac{a^{2}+b^{2}}{2 a b}=\{c, d\}\{e, f\} \text {. }
$$

Thus if one of the progressions is $\{p, q\}, a / b,\{r, s\}$, then the common ratio of the other progression is $(p r+q s) /(p s-q r)$. This shows the reciprocal relationship between the two progressions, and affords a simple method of calculating one from the other.

In this special case, we can extend the configuration of Figure 1 to that of Figure 5, in which all the joins are rational, including those to the point of contact of the circle. So if we invert this configuration in a circle centred at this point of contact, we obtain a configuration of the same form as that of Figure 2. However, although each of the pair of triads of Pythagorean ratios in geometric progression gives rise to configurations of the forms of Figures 1 and 2 related as in Section 6, inversion takes each of these Figure 1 configurations into the non-corresponding Figure 2 configuration.

The simplest example of this special case has $a / b=15 / 8, c / d=26 / 7$ and $e / f=$ $190 / 99$. The arithmetic progression $\{5,2\},\{15,8\},\{4,3\}$ is the case $m=2, n=5$ of the

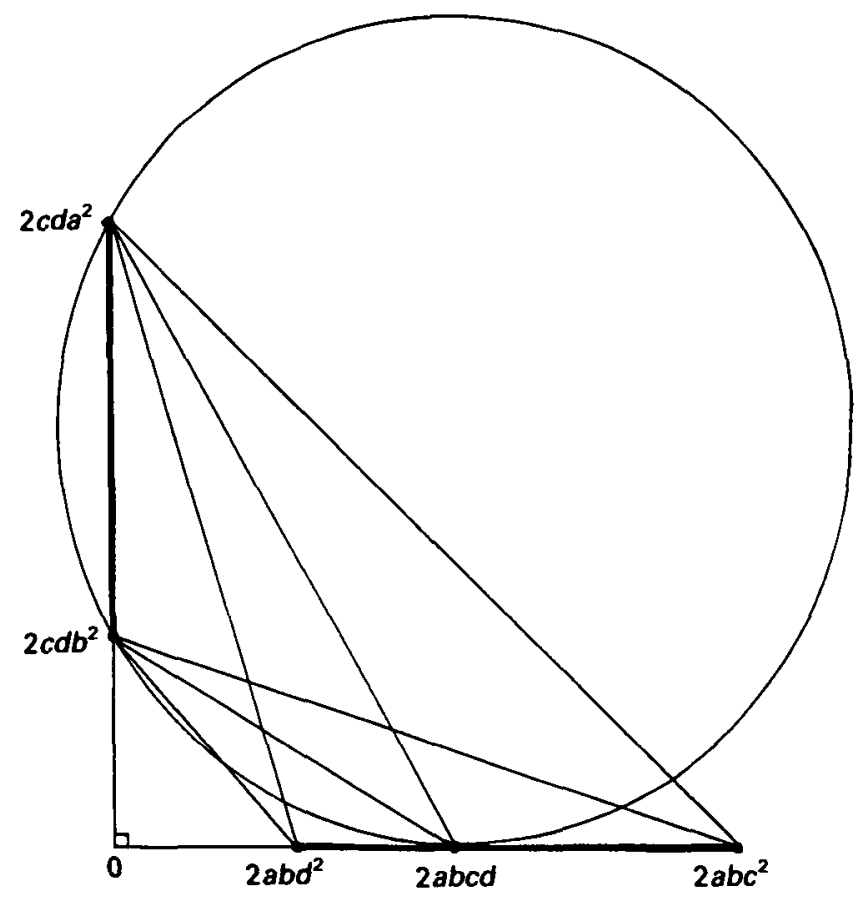

Figure 5. 
progression $\{n, m\},\{n(2 m+n), m(m+2 n)\},\{m+2 n, 2 m+n\}$ given in Section 9 . Other examples can be deduced from solutions of $\{t, u\}^{2}=\{p, q\}\{r, s\}$, of which infinite families and numerical examples are given in [4]. Indeed, if we solve $(n(2 m+n))^{2}+$ $(m(m+2 n))^{2}=$ square, we obtain the infinite family of solutions of $\{t, u\}^{2}=\{p, q\}\{r, s\}$ given in [4] which begins with $\{t, u\}=\{4,1\},\{52,17\},\{3247,1560\}, \ldots$ The other parametric solutions given in Section 9 do not lead to infinite families of solutions of $\{t, u\}^{2}=\{p, q\}\{r, s\}$.

\section{REFERENCES}

1. J. H. E. Cohn, Squares in arithmetical progressions. Math. Scand. 52 (1983), 5-19.

2. L. E. Dickson, History of the Theory of Numbers, vol. 2, Diophantine analysis (Carnegie Institute, Washington 1920) 172-174.

3. R. K. Guy, Unsolved Problems in Number Theory (Springer Verlag, New York and Berlin, 1981).

4. J. Lagrange and J. Leech, Two triads of squares. Maths. Comp. 46 (1986), 751-758.

5. J. Leech, The rational cuboid revisited. Amer. Math. Monthly 84 (1977), 518-533. 